\title{
Thrombus Attached to the Left Atrial Septal Pouch Assessed on 3-Dimensional Transesophageal Echocardiography
}

\author{
Hiroshi Kuwaki, MD; Masaaki Takeuchi, MD; Kyoko Kaku, MD; Nobuhiko Haruki, MD; \\ Hidetoshi Yoshitani, MD; Masahito Tamura, MD; Masahiro Okazaki, MD; \\ Haruhiko Abe, MD; Yutaka Otsuji, MD
}

\section{A}

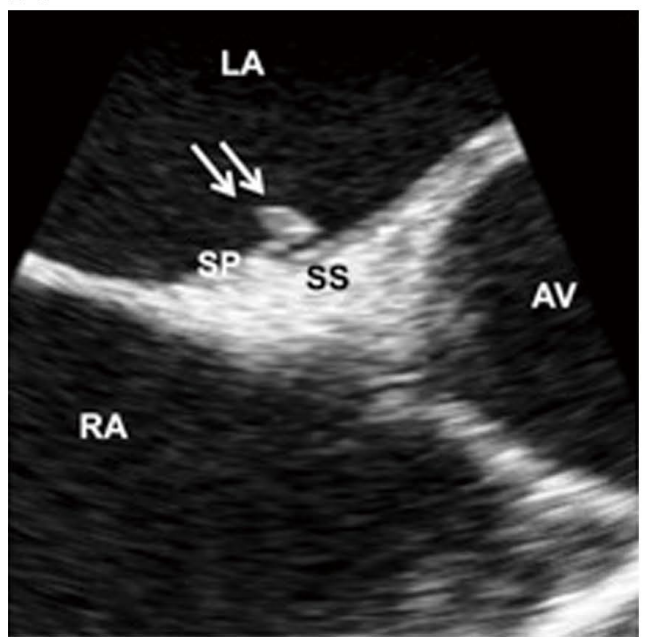

\section{B}
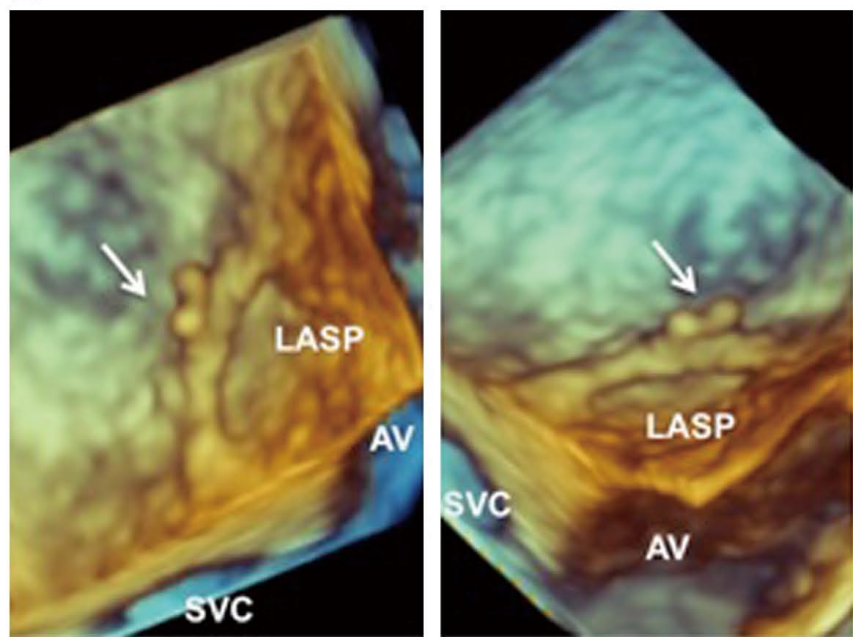

Figure 1. Mass attached to the left atrial septal pouch. (A) Zoomed 2-dimensional (D) transesophageal echocardiogram showing incomplete fusion of septum primum (SP) and septum secundum (SS) creating left atrial septal pouch (LASP). A 6×4-mm mobile mass echo (double white arrows) is attached on the edge of the LASP. (B) Cropped 3-D zoomed images of the interatrial septum clearly depicting LASP and 2 masses (white arrow) attached to the LASP. AV, aortic valve; LA, left atrium; RA, right atrium; SVC, superior vena cava.

$\mathbf{R}$ ecently, left atrial septal pouch (LASP), a kangaroo pouch-like structure on the left atrial side of the interatrial septum, has gained significant interest as a new anatomical entity with a potential for cardioembolic stroke. ${ }^{1}$

A 52-year-old man with a longstanding history of cardiac murmur was admitted to the municipal hospital with a 1-week history of progressive dyspnea. Electrocardiogram showed normal sinus rhythm and possible left ventricular hypertrophy. Chest X-ray showed cardiomegaly, pulmonary congestion and bilateral pleural effusion. Transthoracic echocardiography indicated severe aortic stenosis (AS) probably due to bicuspid aortic valve and severely depressed left ventricular function (left ventricular ejection fraction: 25\%). He was transferred to University Hospital of Occupational and Environmental Health as a definite surgical candidate for aortic valve replacement after the stabilization of heart failure symptoms. He had not taken anticoagulants. Transesophageal echocardiography (TEE) showed that he had severe AS with the anteroposterior type of bicuspid aortic valve. Although the left atrium was severely dilated with a mild degree of spontaneous echo contrast, there was no thrombus in the left atrial appendage. A small mobile mass was incidentally found to be attached on the inter-atrial septum, where fusion between the septum primum and septum secundum is limited to the

Received January 24, 2011; revised manuscript received April 28, 2011; accepted May 11, 2011; released online June 21 , 2011 Time for primary review: 28 days

Second Department of Internal Medicine, University of Occupational and Environmental Health, School of Medicine, Kitakyushu, Japan Mailing address: Masaaki Takeuchi, MD, FASE, Second Department of Internal Medicine, University of Occupational and Environmental Health, School of Medicine, Iseigaoka, Yahatanishi-ku, Kitakyushu 807-0855, Japan. E-mail: takeuchi@med.uoeh-u.ac.jp

ISSN-1346-9843 doi:10.1253/circj.CJ-11-0090

All rights are reserved to the Japanese Circulation Society. For permissions, please e-mail: cj@j-circ.or.jp 

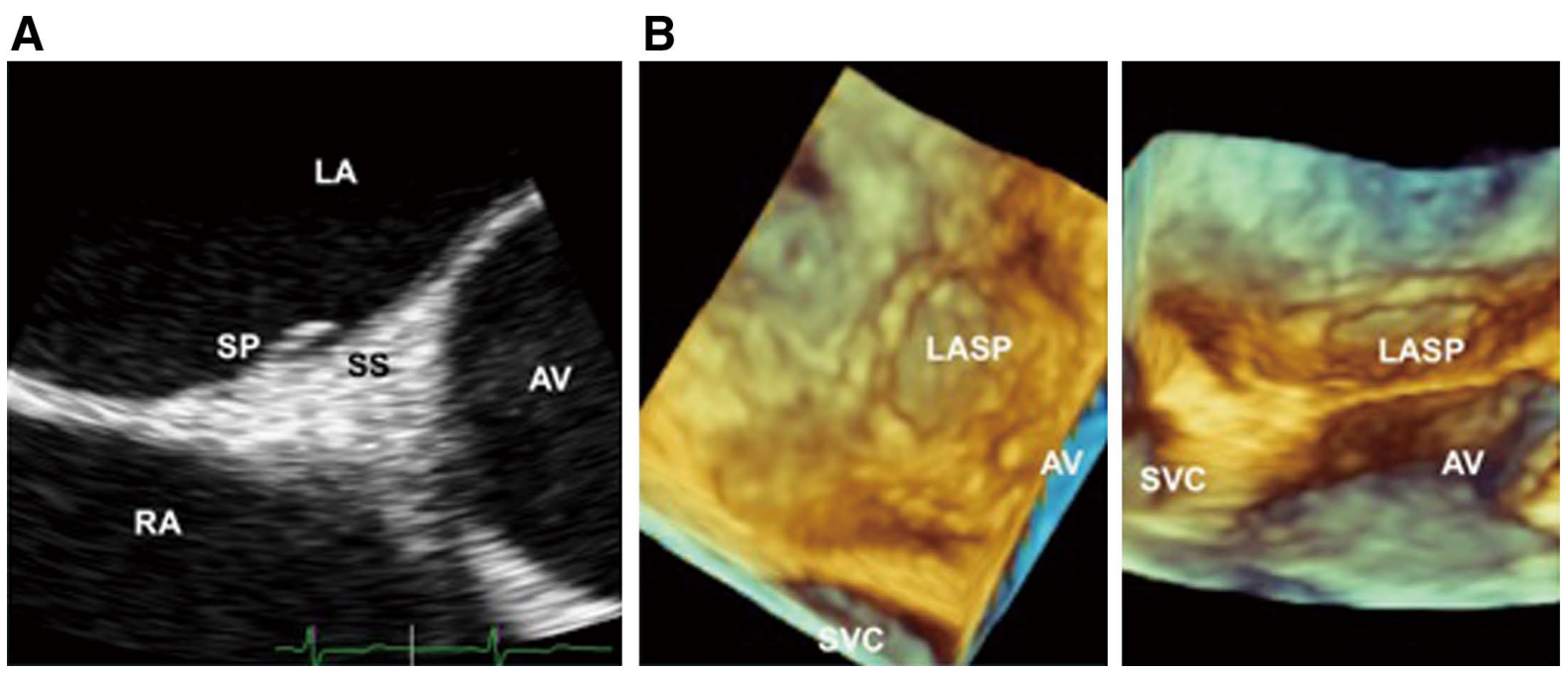

Figure 2. Equivalent (A) 2-dimensional (D) and (B) 3-D images obtained at second transesophageal echocardiography. Masses are no longer visible. AV, aortic valve; LA, left atrium; LASP, left atrial septal pouch; RA, right atrium; SP, septum primum; SS, septum secundum; SVC, superior vena cava.

caudal portion of the zone of overlap resulting in a pouch that can be accessed from the left atrium (LASP). The 3-dimensional (3-D) zoom mode clearly delineated 2 masses attached on the surface of the LASP (Figure 1). Because of the high possibility of thrombus, anticoagulation therapy with warfarin was started. A second TEE at 2 weeks after the initiation of the anticoagulation therapy showed that the masses were no longer visible (Figure 2). During this time the patient did not show any symptoms of systemic embolization, and brain magnetic resonance imaging after the second TEE showed no evidence of recent cerebral infarction.

The LASP is characterized as an incomplete fusion of the septum primum and septum secundum during closure of the foramen ovale. Previous autopsy study has shown that the septum primum and septum secundum fuse only at the caudal limit of the zone of overlap, resulting in a pouch opened to the left atrium in approximately $40 \%$ of subjects. ${ }^{1}$ Because of its anatomical features, the LASP could contribute to blood stasis and thrombus formation. Spontaneous echo contrast due to elevated left ventricular filling pressure observed in the present case reflects blood stasis in the left atrium, which might further exaggerate thrombus formation in the LASP. Diuretic therapy without anticoagulation was also thought to predispose to accelerate thrombus formation. Although we did not directly confirm that masses attached to the LASP are thrombus, disappearance of these masses after the anticoagulation therapy without systemic embolization strongly suggested that they were thrombus. So far, only a few case studies have demonstrated LASP as a site of origin of thrombus formation. ${ }^{2,3}$ Although Tugcu et al recently reported no evidence of the association between the presence of LASP and ischemic or cryptogenic stroke, ${ }^{4}$ their TEE data were obtained between 1997 and 2002, before the concept of LASP was established.

The 2-D transesophageal echocardiography is an established modality for the evaluation of intracardiac mass, but 3D-TEE allows volumetric data acquisition for specific structure, from which the same 2-D cutting plane can be extracted, resulting in accurate and reliable evaluation for the serial change in specific pathologies. ${ }^{5,6}$ As far as we know, this is the first report to describe serial assessment of thrombus attached to LASP, which disappeared after anticoagulation therapy on 2-D- and 3D-TEE. Further prospective study is required to determine the impact of LASP as a potential cause of cardiac embolism. The present case report indicates that more attention is needed to diagnose this structure when TEE is performed, especially in patients in whom cardioembolic stroke is suspected.

\section{References}

1. Krishnan SC, Salazar M. Septal pouch in the left atrium: A new anatomical entity with potential for embolic complications. JACC Cardiovasc Interv 2010; 3: 98-104.

2. Breithardt OA, Papavassiliu T, Borggrefe M. A coronary embolus originating from the interatrial septum. Eur Heart J 2006; 27: 2745.

3. Gurudevan SV, Shah H, Tolstrup K, Siegel R, Krishnan SC. Septal thrombus in the left atrium: Is the left atrial septal pouch the culprit? JACC Cardiovasc Imaging 2010; 3: 1284-1286.

4. Tugcu A, Okajima K, Jin Z, Rundek T, Homma S, Sacco RL, et al. Septal pouch in the left atrium and risk of ischemic stroke. JACC Cardiovasc Imaging 2010; 3: 1276-1283.

5. Iwataki M, Takeuchi M, Yoshitani H, Haruki N, Kaku K, Otsuji Y. Three-dimensional transesophageal echocardiographic recognition of mobile mass protruding into the left main coronary orifice in a patient with aortic stenosis Circ J 2010; 74: 807-808.

6. Haruki N, Takeuchi M, Kaku K, Yoshitani H, Tamura M, Abe H, et al. Prevalence and clinical implication of complex atherosclerotic plaque in the descending thoracic aorta of Japanese patients assessed by transesophageal echocardiography. Circ J 2010; 74: 2627-2632. 